\title{
An Auto-Tuning Damper for the Harmonic Resonance of Grid-Connected Converters
}

\author{
Chia-Tse Lee* Member, Akira Kikuchi* Member \\ Tomomichi Ito* Member
}

(Manuscript received Nov. 5, 2018, revised May 31, 2019)

\begin{abstract}
An auto-tuning damper for grid-connected converters (GCs) is proposed in this paper to improve the stability of GCs when there is an increase in the penetration of renewable energy sources (RES). Increasing number of GCs are being connected to the power grid for supporting the power generation of RES systems, and this makes the equivalent output impedance of GCs becomes closer to the system impedance of the power grid. This increases the influences of the operation of GCs on the output voltage, and there is a greater risk of GCs becoming instable. In addition, system operations such as switching of transmission lines will result in larger variations in the system impedance than before in this situation. This could induce large changes in the resonance characteristics between the system and GCs. In this situation, conventional active damping control, which is designed based on the known conditions of system impedance, could have less controllability due to the changes in resonance characteristics. The harmonic resonance could become instable if the active damping control has insufficient damping effect. For this reason, an auto-tuning damper for harmonic resonance is proposed in this paper. It makes the active damping control capable of taking action in response to the changes in resonance characteristics. Therefore, it can improve the stability of operation of GCs. The operational principle, simulation, and scale-down prototype validation are presented to show the improvements achieved by the proposed solution. In addition, a problem investigated during prototype validation is explained and described.
\end{abstract}

Keywords: power converter, system impedance variations, on-line detection, harmonics, resonances suppression, damping control

\section{Introduction}

Recently, the world has enhanced determination to reduce $\mathrm{CO} 2$ emissions. Many countries have set up plans to increase penetration of renewable energy sources (RES). For example, Japan targets to install $1.7 \%$ of power sources by Wind Power (WP) and $7.0 \%$ of power sources by Photovoltaic (PV) in $2030^{(1)}$, and Germany targets to reach $35 \%$ of the gross electricity consumption by RES in $2020^{(2)}$.

To utilize RES for power generation, grid-connected converters (GCs) are the key equipment to transmit generated power to power grid. In general, output impedance of GCs is relatively larger than the system impedance of power grid when the power capacity of the GCs were relatively much smaller than the short circuit capacity of power grid. In this situation, there is few influences of the operation of GCs on the output voltage at the point that GCs connect to the power grid, because the system impedance of the power grid is low. However, as penetration of RES increases, power capacity of RES becomes comparable to power grid. Increasing number of GCs are being connected in parallel for power generation of RES, so the equivalent output impedance of GCs becomes closer to the system impedance of the power grid. This increases the influences of the operation of GCs on the output voltage at the point that GCs connect to the power grid, which could lead to interferences between the GCs and the

\footnotetext{
* Research and Development Group, Hitachi, Ltd.

7-2-1, Omika-cho, Hitachi, Ibaraki 319-1221, Japan
}

power grid easily. In this situation, system operations such as switching of transmission lines will result in larger variations in the per-unit system impedance, and the system impedance seen from GC affects the stability of $\mathrm{GCs}^{(3)(4)}$, so it should be taken into account when designing the controller of GCs.

In addition, Fault-Ride-Through (FRT) operation has been also required ${ }^{(5)(6)}$. For this reason, dynamic response of the current control of GCs are prone to be designed higher because operation of GCs needs to avoid over-voltage and overcurrent protection tripping during the FRT operation. However, high dynamic response also brings about stability issues, and it might cause harmonic resonance problem. This harmonic resonance problem is highly related to the dynamic characteristics of GCs and the resonance characteristics induced by the inductance and the capacitance components in the output impedance of GCs and the system impedance $^{(3)(4)(7)(8)}$. According to the aforementioned background, it is more difficult to ensure stable operation of GCs, and harmonic resonance problem could occur in GCs when there is an increase in the penetration of RES. These reasons motivate development of solutions for harmonic resonance issue of GCs.

Active damping controls have been widely used for suppressing harmonic resonance in the control of $\mathrm{GCs}^{(9)-(15)}$, and these active damping controls were conventionally designed based on the known conditions of the power grid, that is, there is not too much changes in the resonance characteristic of the power grid. However, since there is larger variations in the system impedance when there is an increase in 
the penetration of RES, the resonance characteristic like resonance frequency could be changed as a result. In this situation, active damping control with constant coefficient could have less controllability for suppressing harmonic resonance. If the active damping control has insufficient damping effect, resonance could become instable and diverge, and this lead to the stop of operation of GCs. Sudden stop of GCs will be stressful to the stability of the power grid when the total power capacity of GCs is comparable to the power capacity of the power grid, and it will be a concern for power system operator. The sudden stop of GCs also causes unexpected losses for power generation business.

In order to avoid harmonic resonance issue deteriorating the stability of the power grid and prevent the unexpected losses for power generation business, an auto-tuning damper for suppressing harmonic resonance is proposed to on-line identify occurrence of resonance and detect its resonance frequency for on-line tuning control coefficient. This method identifies the occurrence of resonance problem quickly, which makes the active damping control start to suppress the resonance before it becomes too high. This prevents the stop of operation of GCs. The operational principles are explained, simulations and scale-down prototype validations are presented in this paper to show the effectiveness of the proposed solution.

\section{System Configuration}

Figure 1 shows a single-line diagram of discussed system of GC, where a converter is being connected to power grid via system impedance. Two factors are considered for the system impedance. One is grid impedance, which is represented by inductance and resistance to emulate the power grid behavior. Another is Power Factor Correction (PFC) filter, which is usually installed for supporting grid voltage and improving voltage quality of the power grid. In GC, a power stage with LC filter is being connected to the power grid via an isolation transformer. In applications of PV and WP generation, power capacity of the GCs typically ranges from several hundreds of kilowatts to a couple of megawatts. In this paper, several hundreds of kilowatts GC is taken as a target for evaluation.

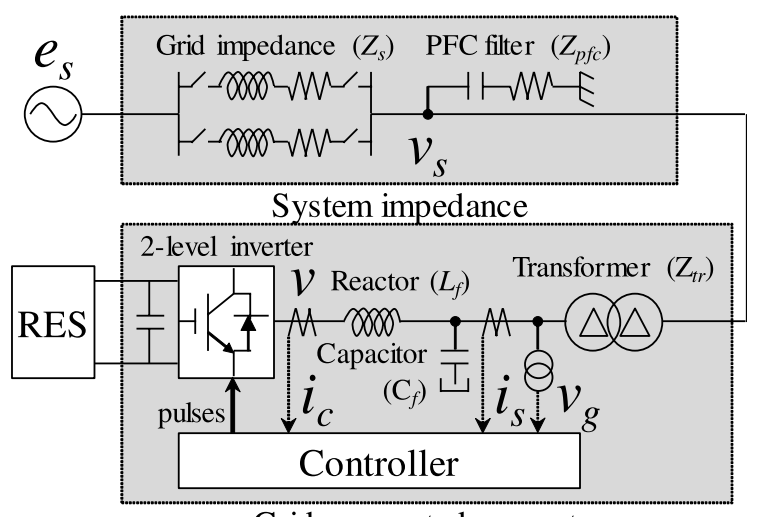

Grid-connected converter

Fig. 1. Single-line diagram of grid-connected converter system

Output voltage and current are sensed and fed back to generate pulses for operating GC by controller. Figure 2 shows the proposed control block of grid-connected converter. Current regulator is implemented by a proportional and integral (PI) control and a voltage feed-forword. The proposed autotuning damper is added before the block of pulse-width modulation (PWM).

A scale-down system is implemented for this study by referring a GC of power capacity of $700 \mathrm{kVA}$. For this purpose, parameters of the GC of power capacity of $700 \mathrm{kVA}$ are designed at first, and then parameters of scale-down system are determined by using the same per-unit value in the referred $700 \mathrm{kVA}$ system. Figure 3 shows the test bench, and Table 1 shows the specifications of the scale-down system.

\section{Auto-tuning Damper}

In this section, the proposed auto-tuning damper is described and explained. As shown in Fig. 2, the proposed autotuning damper is composed of resonance detection and resonance damping, which are explained as follows.

3.1 Resonance Detection Method The proposed auto-tuning damper mainly relies on the resonance detection, whose operation is accomplished by monitoring converter's current $\left(i_{c}\right)$. The resonance detection identifies occurrence of

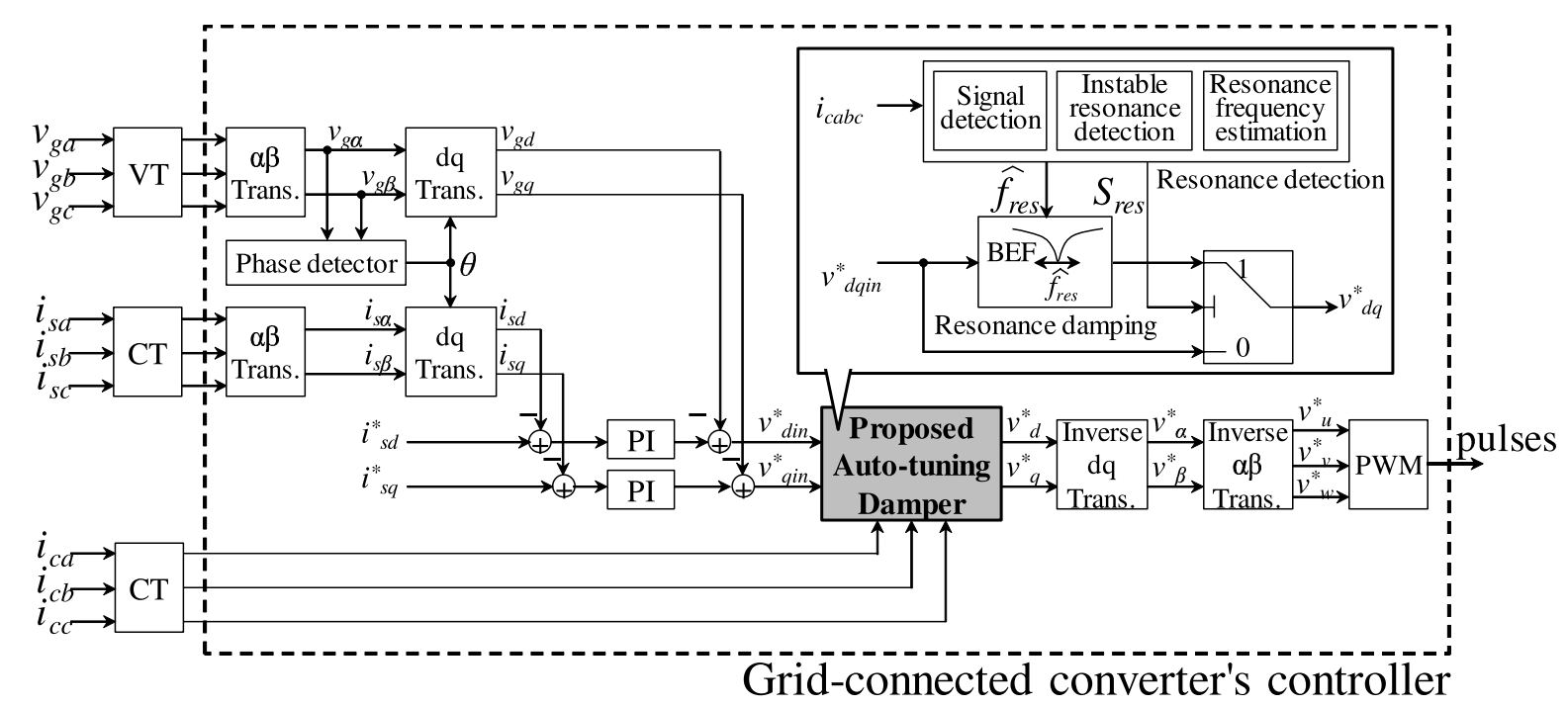

Fig. 2. Overall control block of converter's controller 

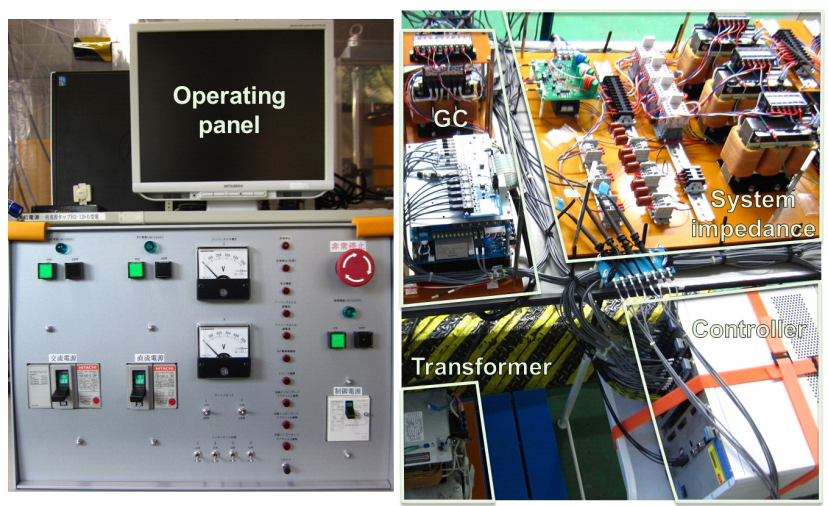

Fig. 3. Scale-down test bench of discussed system

Table 1. Scale-down system

\begin{tabular}{lll}
\hline \hline & \multicolumn{2}{c}{ Power grid } \\
Variables & Symbol & Value \\
\hline Grid voltage & $e_{s}$ & $69 \mathrm{~V}-\mathrm{L} / 50 \mathrm{~Hz}$ \\
Grid impedance & $z_{s}$ & $30 \%(35 \mathrm{mH} / 690 \mathrm{~m} \Omega)$ \\
& & $15 \%($ Two $30 \%$ reactors in parallel) \\
PFC filter & $z_{p f c}$ & $3 \mu \mathrm{F}($ Three $1 \mu \mathrm{F} / 5 \mathrm{~m} \Omega$ capacitors in parallel) \\
\hline \hline & \multicolumn{2}{c}{ Grid-connected converter } \\
Variables & Symbol & Value \\
\hline Rated power & $P_{\text {rated }}$ & $130 \mathrm{~W}$ \\
LC filter & $L_{f}$ & $8.6 \mathrm{mH} / 291 \mathrm{~m} \Omega$ \\
& $C_{f}$ & $1.35 \mu \mathrm{F} / 5 \mathrm{~m} \Omega$ \\
Transformer & $L_{t r}$ & $8.0 \mathrm{mH}$ \\
& $R_{t r}$ & $1053 \mathrm{~m} \Omega$ \\
\hline \hline & \multicolumn{3}{c}{$\mathrm{Controller}$} \\
Variables & Symbol & Value \\
\hline PWM frequency & $f_{s w}$ & $4 \mathrm{kHz}$ \\
Calculation period & $T_{\text {cont }}$ & $125 \mu \mathrm{sec}$ \\
PI controller & $K_{P}$ & $25.8 \mathrm{~V} / \mathrm{A}$ \\
& $K_{I}$ & $873 \mathrm{~V} / \mathrm{A} \cdot \mathrm{sec}^{-1}$ \\
\hline \hline
\end{tabular}

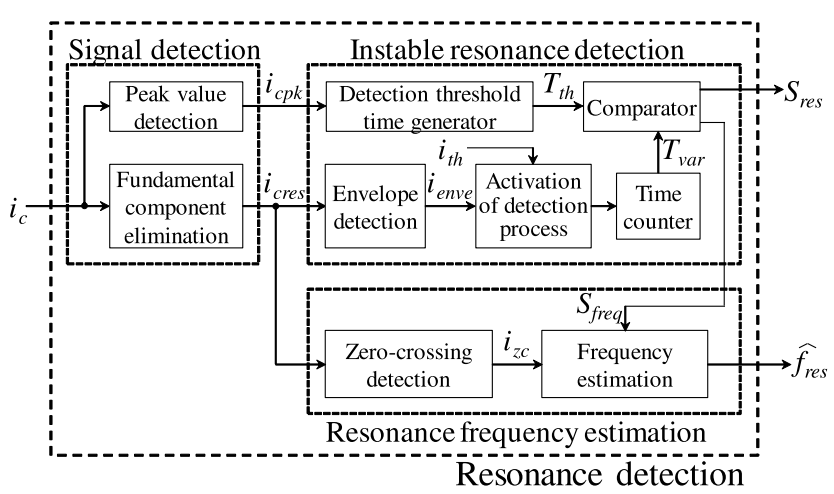

Fig. 4. Control block of proposed resonance detection method

harmonic resonance. When it detects harmonic resonance, it changes a signal flag $\left(S_{\text {res }}\right)$ from 0 to 1 . At the same time, it updates the estimated value to the control coefficient $\left(\hat{f}_{\text {res }}\right)$ in the resonance damping control.

Figure 4 shows control block of proposed resonance detection method, and it contains three main parts, signal detection, instable resonance detection, and resonance frequency estimation. Figure 5 shows the conceptual waveform explaining operational principles of the proposed resonance detection method. As the harmonic resonance happens, resonance oscillation occurs and distorts the converter's current $\left(i_{c}\right)$. Fundamental current component is then eliminated
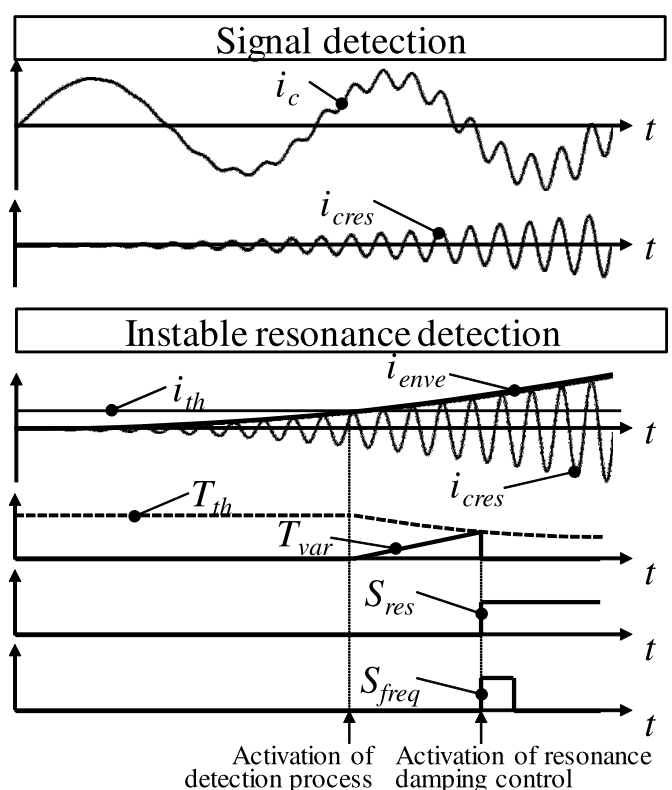

Resonance frequency estimation

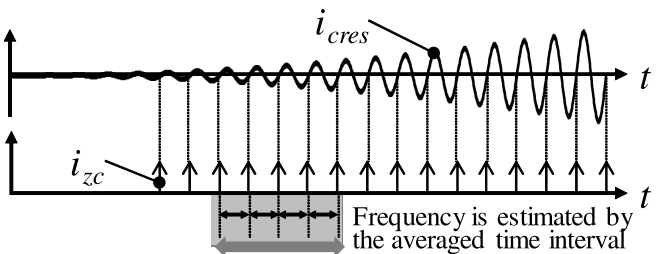

Fig. 5. Conceptual illustration of proposed resonance detection method

by filtering techniques, and the distorted current is derived $\left(i_{\text {cres }}\right)$. At the same time, peak value of the converter's current $\left(i_{c p k}\right)$ can be also detected by monitoring the converter's current $\left(i_{c}\right)$. The proposed resonance detection uses the distorted current $\left(i_{\text {cres }}\right)$ and the peak value of converter's current $\left(i_{\text {cpk }}\right)$ for instable resonance detection and resonance frequency estimation.

Instable resonance detection is developed by monitoring envelope waveform of the distorted current $\left(i_{\text {cres }}\right)$. For this purpose, envelope detection is adopted to derive envelope waveform of the distorted current $\left(i_{\text {enve }}\right)$. When magnitude of envelope becomes higher, it represents that harmonic current increases. Identification of harmonic resonance is developed based on the concept of delay time detection. As shown in Fig. 4, detection process will be activated by comparing the envelope of distorted current $\left(i_{\text {enve }}\right)$ and a pre-determined detection threshold value $\left(i_{t h}\right)$. If the envelope value exceeds the threshold value, detection process begins and a time counter starts to record a detection time $\left(T_{v a r}\right)$. Once the detection time $\left(T_{v a r}\right)$ becomes larger than a detection threshold time $\left(T_{t h}\right)$, the proposed method takes the situation as occurrence of harmonic resonance. In this situation, damping control enable flag $\left(S_{\text {res }}\right)$ is changed for activating active damping control, and damping control coefficient updating flag $\left(S_{\text {freq }}\right)$ is generated for updating value to the control coefficient in active damping control.

Detection threshold value $\left(i_{t h}\right)$ affects sensitivity of proposed resonance detection method, and it can be designed by referring the allowable harmonic resonance current. In this 


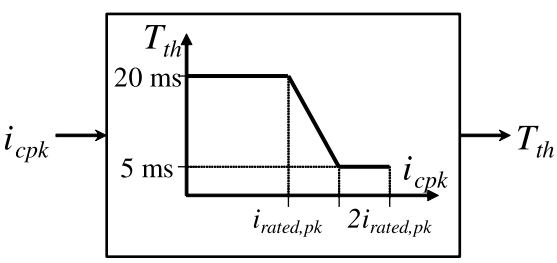

Fig. 6. Detection threshold time generator

paper, the pre-determined detection threshold value was set at 0.1 pu of the rating current of GCs for testing the effectiveness of proposed resonance detection method.

In the proposed resonance detection method, variable detection threshold time is developed to identify the occurrence of resonance detection faster. Figure 6 shows an example for generating the detection threshold time $\left(T_{t h}\right)$ in Fig. 4 . In general, detection threshold time $\left(T_{t h}\right)$ is designed to be smaller as peak value of the distorted current $\left(i_{c p k}\right)$ increases, because there is higher risk of current protection tripping when peak value of the distorted current $\left(i_{c p k}\right)$ increases. For this reason, detection threshold time $\left(T_{t h}\right)$ in Fig. 4 is kept as a normal value when peak value of the distorted current $\left(i_{c p k}\right)$ is lower than rated current. The normal value of detection threshold time should be designed larger than response time of current control for preventing malfunction. On the other hand, when peak value of the distorted current $\left(i_{c p k}\right)$ increases and becomes higher than rated current, the detection threshold time $\left(T_{t h}\right)$ is decreased to make the resonance detection faster since margin of over-current protection decreases, which makes converter have higher risk of protection tripping. In this paper, the normal value is designed as 20 milliseconds in Fig. 6.

During the detection process, the proposed method also estimates resonance frequency by using the zero-crossing detection. It is implemented by detecting zero-crossing points of the distorted current $\left(i_{\text {cres }}\right)$. Time intervals between all the zero-crossing time instances $\left(i_{z c}\right)$ are detected, and the resonance frequency is estimated by averaging all the time intervals. As the instable resonance detection identifies the occurrence of harmonic resonance, it changes the damping control coefficient updating flag $\left(S_{\text {freq }}\right)$, and the estimated resonance frequency is updated to the damping control coefficient $\left(\hat{f}_{\text {res }}\right)$.

In the proposed resonance detection method, zero-crossing technique is adopted for resonance frequency estimation with the assumption that there is only one resonance frequency component as the first step. The proposed method is designed to cope with the instable resonance harmonic problem, so it detects the resonance frequency based on the output waveform when instable situation just happens. The output waveform at this moment would be mainly determined by dominating resonance frequency component, even thought there are multiple resonance frequency components. For this reason, the proposed method detects the dominate component and deal with it as a first priority. The estimation could have error if there are other resonance frequency components, and this issue should be investigated carefully in the future.

3.2 Resonance Damping Method Active damping control methods had been widely proposed and discussed $^{(9)-(15)}$. In this paper, active damping control based on Band-Elimination Filter (BEF) ${ }^{(11)(12)}$ is adopted for validating the proposed resonance detection method. It is implemented
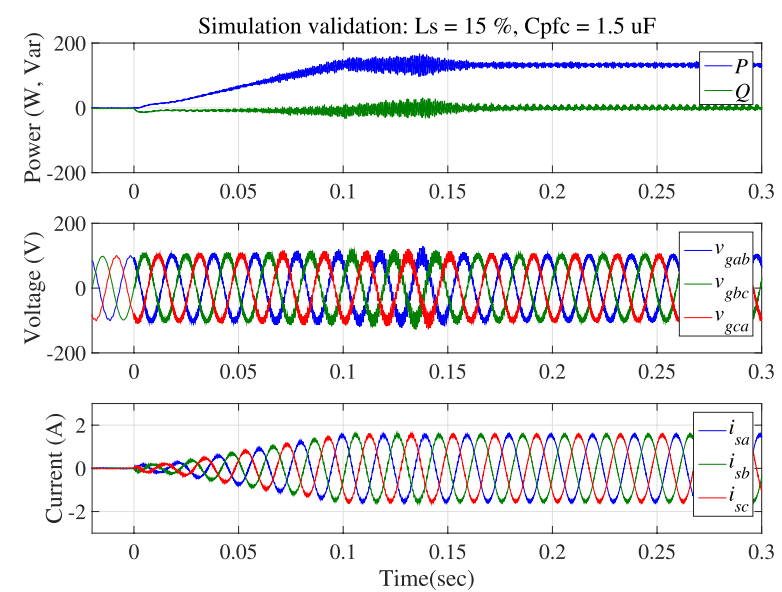

(a) Voltage and current waveform
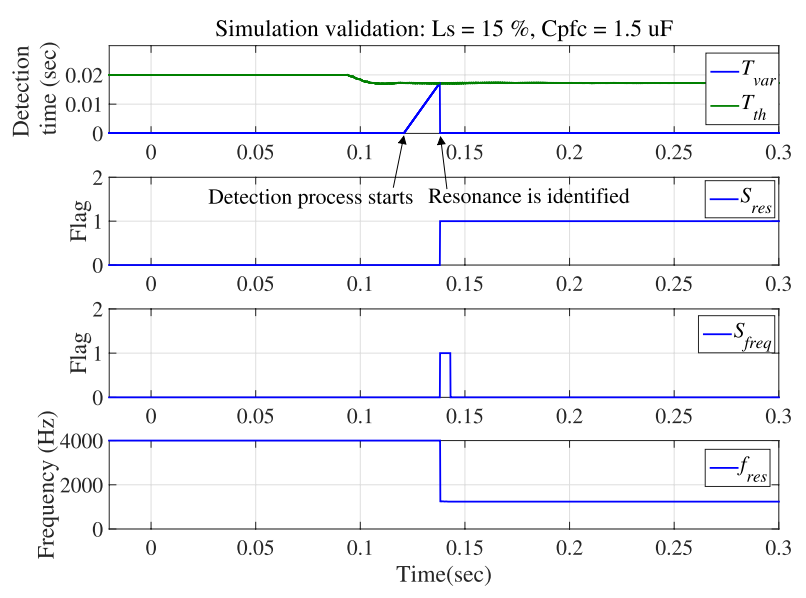

(b) Internal variables in GC's controller

Fig. 7. Simulation validation of auto-tuning damper

as equation (1) shows.

$$
\frac{v_{d q}^{*}}{v_{d q i n}^{*}}= \begin{cases}\frac{s^{2}+4 \pi^{2} \hat{f}_{\text {res }}^{2}}{s^{2}+\frac{2 \pi \hat{f}_{r e s}}{Q}+4 \pi^{2} \hat{f}_{\text {res }}^{2}} & \text { when } S_{\text {res }}=1 \\ 1 & \text { when } S_{\text {res }}=0\end{cases}
$$

As Fig. 2 and equation (1) shows, resonance damping method receives the damping control enable flag $\left(S_{\text {res }}\right)$ to determines whether enabling BEF or not. The eliminated frequency of BEF is adjusted by the estimated resonance frequency $\left(\hat{f}_{r e s}\right)$, and the quality factor $(Q)$ is set as 0.5 in this paper. BEF is adopted as one of resonance damping method in this paper, please note it does not the only active damping control that can work with the resonance detection method.

3.3 Simulation Results According to the description in this section, a time-domain simulation was accomplished based on the system shown in Fig. 1, Fig. 2, Fig. 4, and Fig. 6. Figure 7 shows the results of startup operation when grid impedance is $15 \%$. It shows that harmonic resonance occurred after the operation of GC started and it ramped up its output power. Due to the increase of harmonic resonance, the auto-tuning damper detected the resonance and updated the coefficient of active damping control. Therefore the resonance was suppressed. 


\section{Prototype Validation}

In previous section, operational principle is presented and a preliminary simulation results are presented for validation. In this section, details of the proposed auto-tuning damper was validated in the scale-down prototype shown in Fig. 3. At first, a problem that no harmonic resonance occurs in the prototype hardware was found, so investigation for this problem was done. After the investigation, it was found out that the extra power losses in transformer, system impedance, and LC filter of the prototype were the reason resulting in the differences between prototype hardware and simulation. In previous simulation, as shown in Fig. 7, power losses in the prototype is calculated based on the name plate values, which are derived in the voltage condition of $200 \mathrm{~V}$. Therefore, efficiencies of transformer, system impedance, and LC filter were lower than name plate values because operating voltage in prototype is lower than their name plate efficiencies. Please note that these losses in several hundred kilowatt system will be smaller, so parameter settings in the previous simulation is reasonable. As a result, two cycles of CPU calculation delay were added before the block of inverse $\mathrm{d}-\mathrm{q}$ transformation in the controller of $\mathrm{GC}$ to make harmonic resonance occur easily. This modification could happen in the real system if GC has lower PWM frequency. With this modified setting, the proposed auto-tuning damper was verified in this scaledown prototype. Details about the prototype validation are described in the following subsections.

\subsection{Problem and Cause Investigation At first, the} same scenario as simulation results in Fig. 7 was tested in the prototype, and it was found that harmonic resonance did not occur. For this situation, differences between prototype and settings in the previous simulation were investigated.

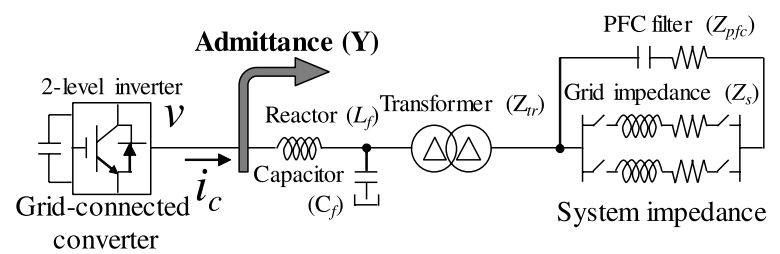

Fig. 8. Configuration for measuring admittance characteristics of prototype
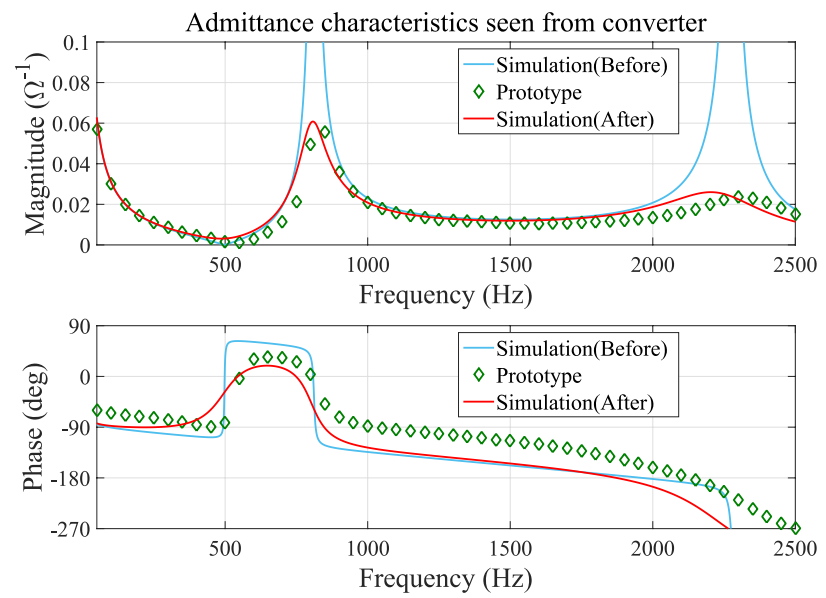

Fig. 9. Investigation on admittance characteristics of prototype
Additional power losses in transformer and impedance could be one possibility, because voltage in the prototype are lower than their name plate values, and these additional power losses in the prototype naturally damped the harmonic resonance. In order to verify this assumption, system admittance seen from GC was measured based on the configuration shown in Fig. 8. Open-loop voltage at different frequencies was injected by using GC, and the output voltage and the current were measured for calculating the admittance seen from GC. As a result, admittances at difference frequencies were plotted in Fig. 9. Blue line in Fig. 9 showed two very high resonance peaks in the admittance characteristics of previous simulation adopted in Section 3.3. However, real admittance characteristic measured in the prototype showed that resonance peaks were much lower than the characteristics of original simulation model. In previous simulation, power losses in transformer, system impedance, and LC filter were derived based on their name plate values and were not estimated accurately. For this reason, power losses in the prototype were estimated and revised in the simulation model. Admittance characteristic of revised simulation model was shown as the red line in Fig. 9, and it became consistent to the admittance characteristic of prototype.

According to the above investigation, it was difficult to create harmonic resonance naturally in the prototype. For

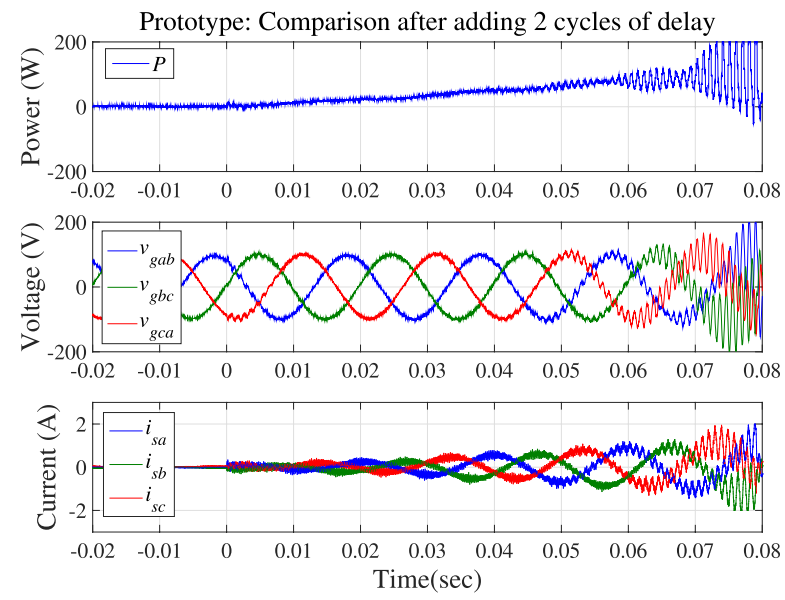

(a) Prototype waveform after adding two cycles of delay block
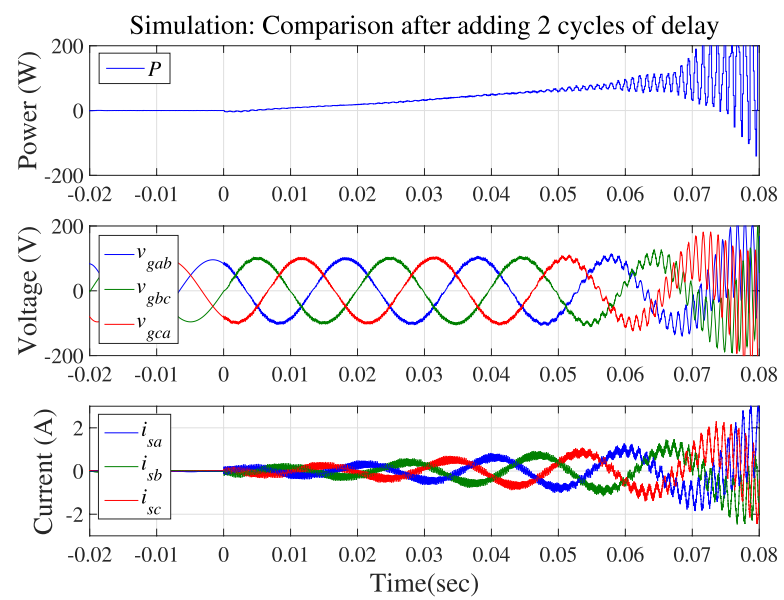

(b) Simulation waveform after adding two cycles of delay block

Fig. 10. Prototype and simulation waveforms after adding two cycles of CPU calculation delay without autotuning damper 

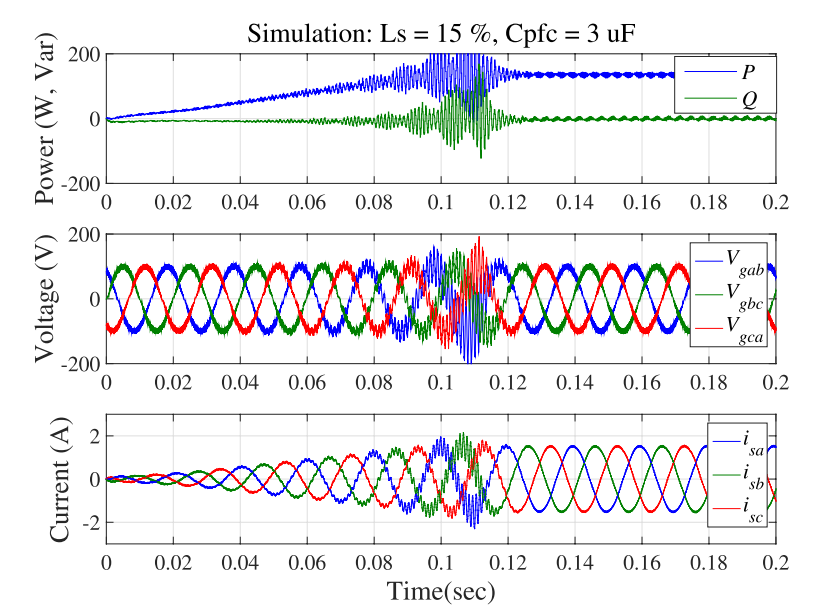

(a) Magnified waveform
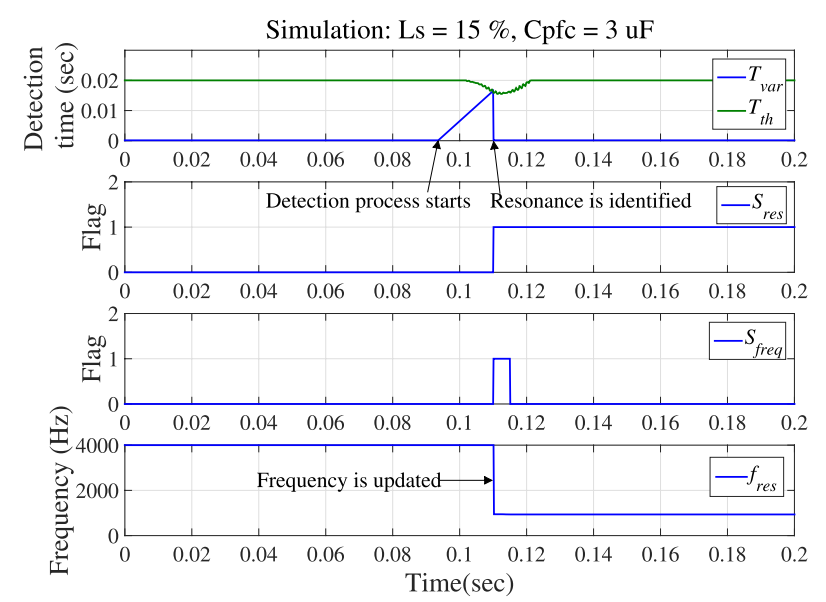

(b) Internal variables in GC's controller

Fig. 11. Simulation results when grid impedance is $15 \%$

this reason, two cycles of CPU calculation delay were added before the block of inverse d-q transformation in the controller of GC, Fig. 2, to make the harmonic resonance occur easily. This modification could happen in the real system if GC has lower PWM frequency. After this modification, harmonic resonance occurred in the prototype, and Fig. 10 showed the prototype and the revised simulation waveforms without auto-tuning damper. Similar results were derived, and harmonic resonance occurred after converter started to operate. In Fig. 10(a), the protection of GC was tripped by harmonic resonance and then the operation of GC was stopped at around 0.08 second.

4.2 Final Validation with Prototype The proposed auto-tuning damper were tested in the prototype with the aforementioned two cycles of CPU calculation delay. Corresponding simulation results are also presented for comparison. Figure 11 and 12 show converter's startup operation with simulation and prototype when the grid impedance is $15 \%$. Figure 11(a) shows the output power, voltage, and current of GC and Fig. 11(b) is the internal variables in the proposed auto-tuning damper during the occurrence of harmonic resonance. In Fig. 11, converter ramped up its active power at 0 second, and the harmonic resonance occurred and increased. Due to the occurrence of harmonic resonance, the proposed auto-tuning damper started resonance detection at $0.095 \mathrm{sec}-$ ond and identified the resonance at 0.11 second. As shown in
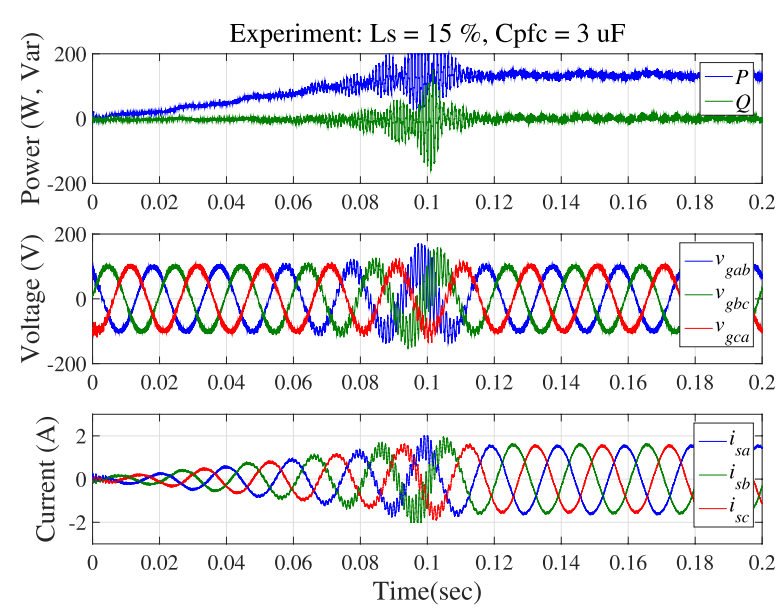

(a) Magnified waveform

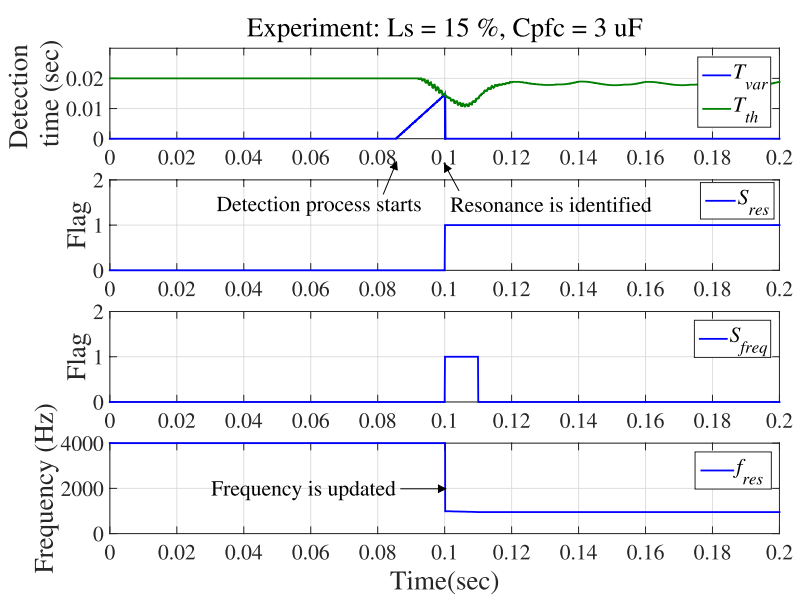

(b) Internal variables in GC's controller

Fig. 12. Experimental tests when grid impedance is $15 \%$

Fig. 11(b), enable flag changed to activate resonance damping and estimated frequency for resonance damping was also updated after the auto-tuning damper detected the resonance. Therefore, the resonance was suppressed and the stable operation of GC continued. The same operation can be also observed in the experimental results shown in Fig. 12.

Figures 13 and 14 show operations in simulation and prototype when grid impedance is changed from $15 \%$ to $30 \%$. Figure 13(a) shows output power, voltage, and current of GC. It shows that GC rode through startup operation and outputted rated power stably at first, and then grid impedance changed from $15 \%$ to $30 \%$ at -0.125 second. Harmonic resonance thus gradually occurred again because characteristic of harmonic resonance changes due to the variations in grid impedance. In response to the harmonic resonance, the proposed auto-tuning damper started the detection process again at -0.02 second, and it identified the resonance at 0 second as shown in Fig. 13(b). By updating the frequency for the damping control again, the harmonic resonance was suppressed. The same operation in prototype was also tested and shown in Fig. 14.

\section{Discussion}

In this paper, a scale-down prototype was built for validating the proposed auto-tuning damper. However, power losses in prototype were high, so harmonic resonance was damped 
Table 2. Simulation comparisons for discussion

\begin{tabular}{|c|c|c|c|c|}
\hline Simulation conditions & $\begin{array}{l}\text { Simulation with parameters } \\
\text { calculated based on name plate } \\
\text { values in prototype (Fig. 7) }\end{array}$ & $\begin{array}{l}\text { Simulation emulating prototype } \\
\text { settings in this paper } \\
\text { (Fig. 10(b), Fig. 11, Fig. 13) }\end{array}$ & $\begin{array}{l}\text { Simulation based on the target } \\
700 \text { kVA system with typical } \\
\text { transformer settings (Fig. 15) }\end{array}$ & $\begin{array}{l}\text { Simulation based on the target } 700 \mathrm{kVA} \\
\text { system with transformer parameters } \\
\text { measured in prototype (Fig. 16) }\end{array}$ \\
\hline Power capacity $\left(P_{\text {rated }}\right)$ & $130 \mathrm{VA}$ & $130 \mathrm{VA}$ & $700 \mathrm{kVA}$ & $700 \mathrm{kVA}$ \\
\hline Voltage $\left(e_{s}\right)$ & $69 V_{L-L}$ & $69 \mathrm{~V}_{\mathrm{L}-\mathrm{L}}$ & $6.6 \mathrm{kV}_{\mathrm{L}-\mathrm{L}}$ & $6.6 \mathrm{kV}_{\mathrm{L}-\mathrm{L}}$ \\
\hline Transformer $\left(V_{1} / V_{2}, X_{t r}, R_{t r}\right)$ & $69 \mathrm{~V} / 69 \mathrm{~V}, 7 \%, 2.9 \%$ & $69 \mathrm{~V} / 69 \mathrm{~V}, 7 \%, 2.9 \%$ & $360 \mathrm{~V} / 6.6 \mathrm{kV}, 7 \%, 2.9 \%$ & $360 \mathrm{~V} / 6.6 \mathrm{kV}, 7 \%, 2.9 \%$ \\
\hline Transformer $\left(X_{m}, R_{m}\right)$ & $4300 \%, 5100 \%$ & $1700 \%, 1150 \%$ & $20000 \%, 20000 \%$ & $1700 \%, 1150 \%$ \\
\hline Grid impedance $\left(X_{s}\right)$ & $15 \%$ & $15 \%, 30 \%$ & $15 \%$ & $15 \%$ \\
\hline PFC filter $\left(Y_{p f c}\right)$ & $1.72 \%$ & $3.45 \%$ & $1.72 \%$ & $1.72 \%$ \\
\hline Filter reactor $\left(X_{L f}, Y_{C f}\right)$ & $7.5 \%, 1.55 \%$ & $7.5 \%, 1.55 \%$ & $7.5 \%, 1.55 \%$ & $7.5 \%, 1.55 \%$ \\
\hline Additional delay in CPU & 0 & 2 & 0 & 0 \\
\hline
\end{tabular}

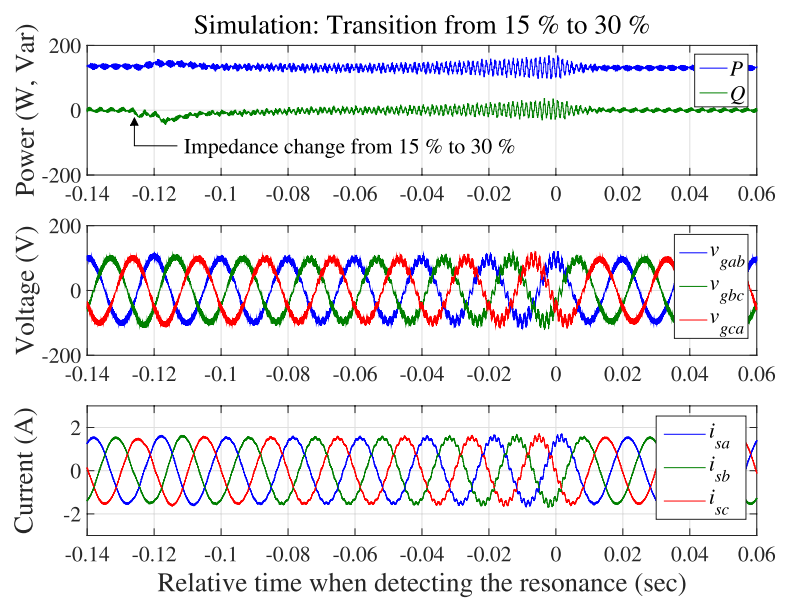

(a) Magnified waveform
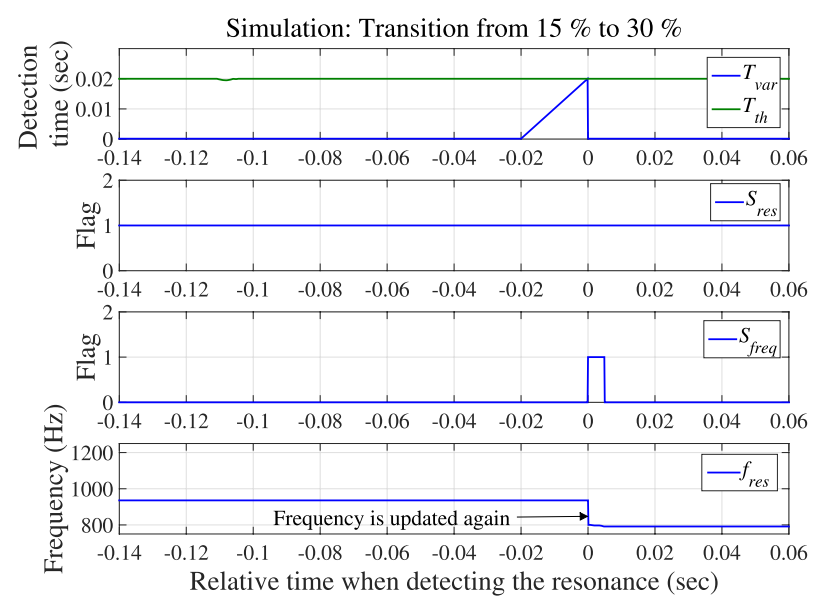

(b) Internal variables in GC's controller

Fig. 13. Simulation results when grid impedance change from $15 \%$ to $30 \%$

naturally. Therefore, two cycles of CPU calculation delay were added in the controller of GC to make the harmonic resonance occur easily in the prototype. It should be discussed to justify that the similar phenomenon could still happen in the real system. For this reason, several simulations are provided to proof it. Table 2 compares parameters in different simulations.

Before building the scale-down prototype, simulation was confirmed with parameters calculated according to their name plate values. Simulation results presented in Fig. 7 showed that harmonic resonance occurred, and the proposed auto-tuning damper was effective to suppress the harmonic resonance. In addition, CPU calculation delay was not added in this case. However, real magnetizing inductance and re-
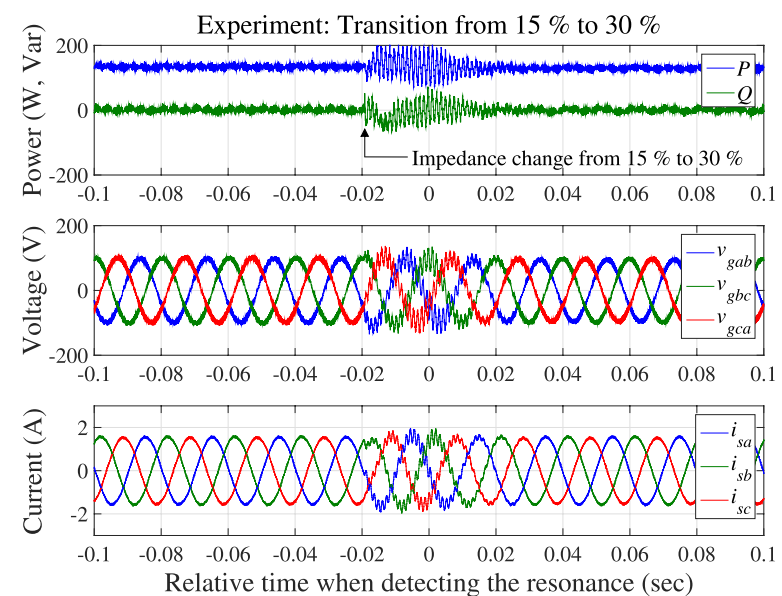

(a) Magnified waveform
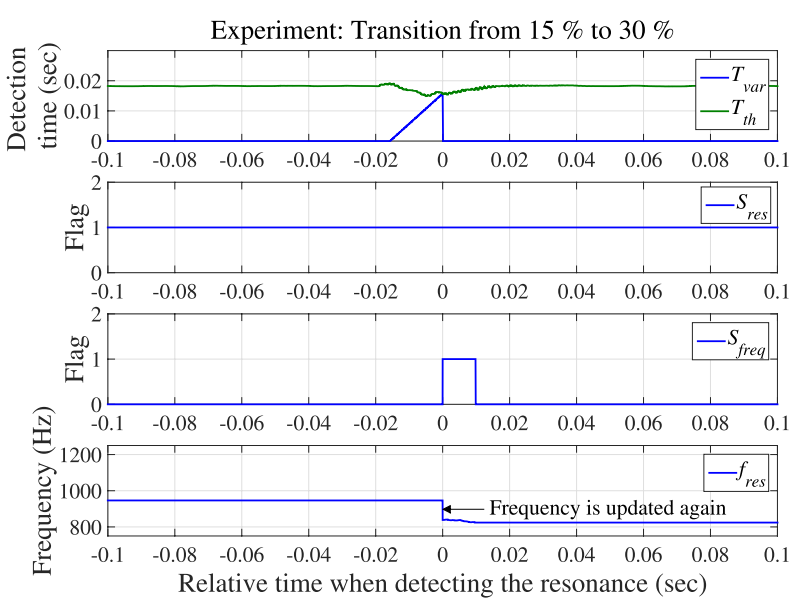

(b) Internal variables in GC's controller

Fig. 14. Experimental tests when grid impedance change from $15 \%$ to $30 \%$

sistance in the transformer were found out lower than values calculated from name plate values, because the name plate values were measured at $200 \mathrm{~V}$ operation voltage. For this reason, two cycles of CPU calculation delay are added in the controller of GC, and the proposed auto-tuning damper was verified with this parameter settings. This additional CPU calculation delay is considered to be practical because longer delay could happen if the GC has lower PWM frequency. On the other hand, in order to confirm that harmonic resonance could still occurs in the target system, simulations based on the $700 \mathrm{kVA}$ system with practical settings in the transformer and the controller of GC. In this situation, there is no additional CPU calculation delay, and magnetizing inductance and resistance were both set as $20000 \%$. Figure 15 shows 

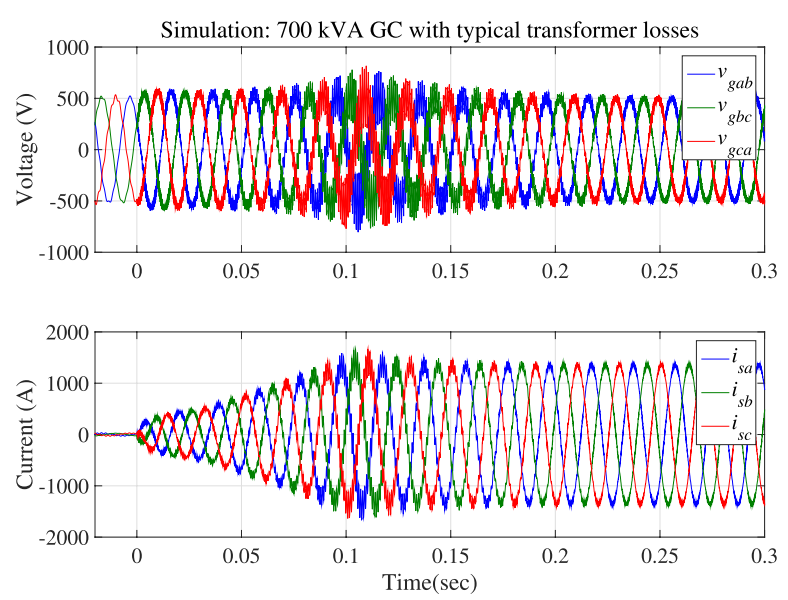

Fig. 15. Simulation of proposed auto-tuning damper in real-scale system with typical transformer losses
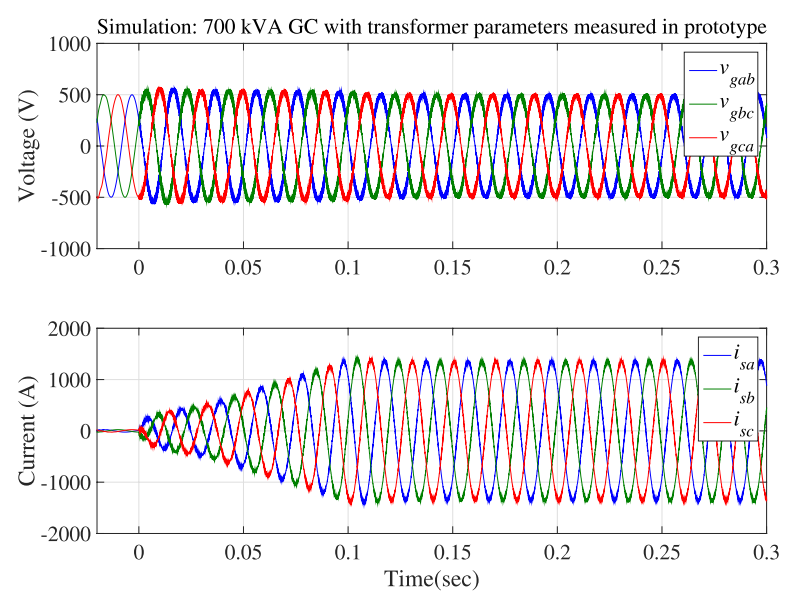

Fig. 16. Simulation of proposed auto-tuning damper in real-scale system with transformer parameters measured in scale-down prototype

that harmonic resonance still occurred and the proposed autotuning damper was effective to suppress the harmonic resonance. In the end, a simulation based on the target $700 \mathrm{kVA}$ system with transformer parameters measured in the prototype was confirmed in Fig. 16, and it was found out that the harmonic resonance was also damped naturally.

\section{Conclusion}

This paper proposes an auto-tuning damper for gridconnected converter (GC). The proposed method on-line identifies the occurrence of harmonic resonance and detects the resonance frequency at the same time. Occurrence of harmonic resonance is identified by monitoring the envelope of harmonic resonance current and the resonance frequency is estimated by using the zero-crossing points of harmonic resonance current.

In order to verify the proposed auto-tuning damper, simulation was accomplished in advance and a scale-down prototype was built for validation. However, it was found that harmonic resonance did not occur in the scale-down prototype. After investigation, it was proven that power losses were higher than expected in the prototype, and they naturally damped harmonic resonance. In response to this issue, controller of GC was modified to make harmonic resonance occur easier, and performance of the proposed auto-tuning damper was verified. According to the validation results, harmonic resonance can be identified within 20 milliseconds after the method starts the detection process. By updating the resonance frequency for the active damping control, GC is capable of riding through wide range changes in the resonance frequency. This improves the stability of operation of GC.

In the end, simulations based on real system was accomplished to confirm that the harmonic resonance phenomenon could still occur in the real system, and the proposed autotuning damper helps suppressing the harmonic resonance.

\section{References}

( 1 ) Ministry of Economy, Trade and Industry: The Long-term Energy Demand and Supply Outlook (2015)

( 2 ) International Energy Agency, IEA: IEA-PVPS Annual Report 2015 (2015)

( 3 ) M. Liserre, R. Teodorescu, and F. Blaabjerg: "Stability of Photovoltaic and Wind Turbine Grid-Connected Inverters for a Large Set of Grid Impedance Value", IEEE Transactions on Power Electronics, Vol.21, No.1, 263-272 (2006)

( 4 ) J. Sun: "Impedance-based Stability Criterion for Grid-connected Inverters", IEEE Transactions on Power Electronics, Vol.26, No.11, 3075-3078 (2011)

( 5 ) R. Teodorescu, M. Liserre, and P. Rodriguez: Grid Converters for Photovoltaic and Wind Power Systems, Wiley-IEEE Press (2011)

( 6 ) T. Ito, A. Kikuchi, H. Nemoto, M. Ichinose, and M. Taniguchi: "Enhanced Current Control Scheme for Large-Scale Solar Inverters", PCIM Europe 2017 (2017)

( 7 ) J.B. Glasdam, L.H. Kocewiak, J. Hjerrild, and C.L. Bak: "Control System interaction in the VSC-HVDC Grid Connected Offshore Wind Power Plant", CIGRE Symposium (2015)

( 8 ) M. Liserre, F. Blaabjerg, and R. Teodorescu: "Grid Impedance Estimation via Excitation of LCL-Filter Resonance", IEEE Transactions on Industry Applications, Vol.43, No.5, 1401-1407 (2007)

( 9 ) V. Blasko and V. Kaura: "A Novel Control to Actively Damp Resonance Input LC filter of a Three-phase Voltage Source Converter", IEEE Transactions on Industry Applications, Vol.33, No.2, 542-550 (1997)

(10) H. Akagi, H. Fujita, and K. Wada: "A Shunt Active Filter Based on Voltage Detection for Harmonic Termination of a Radial Power Distribution Line", IEEE Transactions on Industry Applications, Vol.35, No.3, 638-645 (1999)

(11) C. Liu, X. Zhang, L. Tan, and F. Liu: "A Novel Control Strategy of LCLVSC based on Notch Concept", The 2nd International Symposium on Power Electronics for Distributed Generation Systems, 343-346 (2010)

(12) J. Dannehl, M. Liserre, and F.W. Fuchs: "Filter-Based Active Damping of Voltage Source Converters with LCL Filters", IEEE Transactions on Industrial Electronics, Vol.58, No.8, 3623-3633 (2011)

(13) X. Wang, F. Blaabjerg, M. Liserre, Z. Chen, J. He, and Y. Li: "An Active Damper for Stabilizing Power-Electronics-Based AC Systems", IEEE Transactions on Power Electronics, Vol.29, No.7, 3318-3329 (2014)

(14) C. Bao, X. Ruan, X. Wang, W. Li, D. Pan, and K. Weng: "Stepby-Step Controller Design for LCL-Type Grid-Connected Inverter with CapacitorCurrent-Feedback Active-Damping", IEEE Transactions on Power Electronics, Vol.29, No.3, 1239-1259 (2014)

(15) R. Pea-Alzola, M. Liserre, F. Blaabjerg, R. Sebastin, J. Dannehl, and F.W. Fuchs: "Systematic Design of the Lead-Lag Network Method for Active Damping in LCL-Filter Based Three Phase Converters", IEEE Transactions on Industrial Informatics, Vol.10, No.1, 43-52 (2014)

Chia-Tse Lee (Member) received the Ph.D. degrees in electrical engineering from National Tsing Hua University, Hsinchu, Taiwan, in 2013. He is currently a researcher with Research and Development Group, Hitachi, Ltd. His current research interests include high power converters and applications, and power electronic technologies for renewable energy and smart grid. Dr. Lee received the IEEE IAS Committee Prize Paper Awards in 2012 and 2014, respectively. 
Akira Kikuchi (Member) received the B.E. and M.E. degrees in electrical engineering from Kyoto University, Kyoto, Japan, in 1995 and 1997, respectively. He is currently a senior researcher with Research and Development Group, Hitachi, Ltd. His current research interests include high power converters and applications, and power electronic technologies for renewable energy and smart grid.
Tomomichi Ito (Member) received the B.E. and M.E. degrees in electrical engineering from Hokkaido University, Sapporo, Japan, in 1998 and 2000, respectively. He is currently leading a research group consists of 10 researchers at Hitachi, Ltd. He has mainly developed control scheme of high-power grid-connected inverters including inverters for renewable energy sources. 